\title{
MEAN CONVERGENCE THEOREM FOR MULTIDIMENSIONAL ARRAYS OF RANDOM ELEMENTS IN BANACH SPACES
}

\author{
LE VAN THANH
}

Received 2 March 2006; Revised 29 June 2006; Accepted 12 July 2006

For a $d$-dimensional array of random elements $\left\{V_{n}, n \in \mathbb{Z}_{+}^{d}\right\}$ in a real separable stable type $p(1 \leq p<2)$ Banach space, a mean convergence theorem is established. Moreover, the conditions for the convergence in mean of order $p$ are shown to completely characterize stable-type $p$ Banach spaces.

Copyright (c) 2006 Le Van Thanh. This is an open access article distributed under the Creative Commons Attribution License, which permits unrestricted use, distribution, and reproduction in any medium, provided the original work is properly cited.

\section{Introduction}

Let $\mathbb{Z}_{+}^{d}$, where $d$ is a positive integer, denote the positive integer $d$-dimensional lattice points. The notation $m \prec n$, where $m=\left(m_{1}, m_{2}, \ldots, m_{d}\right)$ and $n=\left(n_{1}, n_{2}, \ldots, n_{d}\right) \in \mathbb{Z}_{+}^{d}$, means that $m_{i} \leqslant n_{i}, 1 \leqslant i \leqslant d,|n|$ is used for $\prod_{i=1}^{d} n_{i}$.

Gut [5] proved that if $\left\{X, X_{n}, n \in \mathbb{Z}_{+}^{d}\right\}$ is a $d$-dimensional array of i.i.d. random variables with $E|X|^{p}<\infty(0<p<2)$ and $E X=0$ if $1 \leqslant p<2$, then

$$
\frac{\sum_{j<n} X_{j}}{|n|^{1 / p}} \longrightarrow 0 \quad \text { in } L^{p} \text { as } \min _{1 \leqslant i \leqslant d} n_{i} \longrightarrow \infty,
$$

where $\left(n_{1}, n_{2}, \ldots, n_{d}\right)=n \in \mathbb{Z}_{+}^{d}$.

Recently, Thanh [11] proved (1.1) under condition of uniform integrability of $\left\{\left|X_{n}\right|^{p}\right.$, $\left.n \in \mathbb{Z}_{+}^{d}\right\}$.

Mean convergence theorems for sums of random elements Banach-valued are studied by many authors. The reader may refer to Wei and Taylor [12], Adler et al. [2], Rosalsky and Sreehari [9], or more recently, Rosalsky et al. [10], Cabrera and Volodin [3]. However, we are unaware of any literature of investigation on the mean convergence for multidimensional arrays of random elements in Banach spaces.

Consider a $d$-dimensional array $\left\{V_{n}, n \in \mathbb{Z}_{+}^{d}\right\}$ of independent random elements defined on a probability space $(\Omega, \mathscr{F}, P)$ and taking values in a real separable Banach space 
$\mathscr{L}$ with norm $\|\cdot\|$. In the current work, we establish the convergence in mean of order $p(1 \leqslant p<2)$ of the sums $\sum_{j<n} V_{j} /|n|^{1 / p}, n \in \mathbb{Z}_{+}^{d}$, under the condition that $\left\{\left\|V_{n}\right\|^{p}, n \in\right.$ $\left.\mathbb{Z}_{+}^{d}\right\}$ is uniformly integrable. The main results of this paper are Theorems 2.1 and 2.2. Theorem 2.1 is a stable-type $p$ Banach space version of the main result of Thanh [11]. While the proof of Theorem 2.1 and the proof of the main result in Thanh [11] are similar, we will show in Theorem 2.2 that the implication in Theorem 2.1 indeed completely characterizes stable-type $p$ Banach spaces.

Let $0<p \leqslant 2$ and let $\left\{\theta_{n}, n \geqslant 1\right\}$ be independent and identically distributed stable random variables each with characteristic function $\phi(t)=\exp \left\{-\left|t^{p}\right|\right\}$. The real separable Banach space $\mathscr{X}$ is said to be of stable-type $p$ if $\sum_{n=1}^{\infty} \theta_{n} v_{n}$ converges a.s. whenever $v_{n} \in \mathscr{X}, n \geqslant 1$ with $\sum_{n=1}^{\infty}\left\|v_{n}\right\|^{p}<\infty$. Equivalent characterizations of a Banach space being of stable-type $p$, properties of stable-type $p$ Banach spaces, as well as various relationships between the conditions Rademacher-type $p$, and stable-type $p$ may be found by Woyczyński in [13], by Marcus and Woyczyński in [7], and by Pisier in [8], see also the discussion by Adler et al. in [1]. We now mention explicitly some characterizations of this concept. The first theorem was obtained by Mandrekar and Zinn [6] and by Marcus and Woyczyński [7].

Theorem 1.1. Let $1 \leqslant p<2$ and let $\mathscr{X}$ be a real separable Banach space. Then the following statements are equivalent.

(i) $\mathscr{X}$ is of stable-type $p$.

(ii) For every symmetric random elements $V$, the condition $n^{p} P(\|V\|>n) \rightarrow 0$ as $n \rightarrow$ $\infty$ implies that

$$
\frac{\sum_{j=1}^{n} V_{j}}{n^{1 / p}} \longrightarrow 0 \quad \text { in probability }
$$

where $\left\{V_{j}, j \geqslant 1\right\}$ are independent copies of $V$.

Theorem 1.2 (see [13, Theorem V.9.3]). Let $1 \leqslant p<2$ and let $\mathscr{L}$ be a real separable Banach space. Then the following statements are equivalent.

(i) $\mathscr{X}$ is of stable-type $p$.

(ii) For each bounded sequence $\left\{x_{n}, n \geqslant 1\right\}$ of elements of $\mathscr{X}$,

$$
\frac{\sum_{j=1}^{n} x_{k} \epsilon_{k}}{n^{1 / p}} \longrightarrow 0 \quad \text { a.s. }
$$

where $\left\{\epsilon_{n}, n \geqslant 1\right\}$ is a Rademacher sequence.

The symbol $C$ denotes throughout a generic constant $(0<C<\infty)$ which is not necessarily the same one in each appearance.

\section{Main results}

We can now present the main results. Theorem 2.1 is a stable-type $p$ Banach space version of the main result of Thanh [11]. 
Theorem 2.1. Let $\left\{V_{n}, n \in \mathbb{Z}_{+}^{d}\right\}$ be a d-dimensional array of independent mean-zero random elements in a real separable stable-type $p(1 \leqslant p<2)$ Banach space $\mathscr{X}$. If

$$
\left\{\left\|V_{n}\right\|^{p}, n \in \mathbb{Z}_{+}^{d}\right\} \text { is uniformly integrable, }
$$

then

$$
\frac{\sum_{j \prec n} V_{j}}{|n|^{1 / p}} \longrightarrow 0 \quad \text { in } L^{p} \text { as }|n| \longrightarrow \infty \text {. }
$$

Proof. For arbitrary $\epsilon>0$, there exists $M>0$ such that

$$
E\left(\left\|V_{n}\right\|^{p} I\left(\left\|V_{n}\right\|>M\right)\right)<\epsilon, \quad \forall n \in \mathbb{Z}_{+}^{d}
$$

Set

$$
V_{n}^{\prime}=V_{n} I\left(\left\|V_{n}\right\| \leqslant M\right), \quad n \in \mathbb{Z}_{+}^{d}, \quad V_{n}^{\prime \prime}=V_{n} I\left(\left\|V_{n}\right\|>M\right), \quad n \in \mathbb{Z}_{+}^{d} .
$$

Since $\mathscr{X}$ is of stable-type $p$ and $p<2$, it is of Rademacher-type $q$ for some $p<q<2$. Thus

$$
\begin{aligned}
E\left\|\sum_{j<n} V_{j}\right\|^{p} & \leqslant 2^{p-1}\left[E\left\|\sum_{j<n}\left(V_{j}^{\prime}-E V_{j}^{\prime}\right)\right\|^{p}+E\left\|\sum_{j<n}\left(V_{j}^{\prime \prime}-E V_{j}^{\prime \prime}\right)\right\|^{p}\right] \\
& \leqslant 2^{p-1} E\left\|\sum_{j<n}\left(V_{j}^{\prime}-E V_{j}^{\prime}\right)\right\|^{p}+C \sum_{j<n} E\left\|V_{j}^{\prime \prime}-E V_{j}^{\prime \prime}\right\|^{p} \\
& \leqslant 2^{p-1}\left(E\left\|\sum_{j<n}\left(V_{j}^{\prime}-E V_{j}^{\prime}\right)\right\|^{q}\right)^{p / q}+C \sum_{j<n} E\left\|V_{j}^{\prime \prime}-E V_{j}^{\prime \prime}\right\|^{p}
\end{aligned}
$$

(by the Jensen inequality)

$$
\begin{aligned}
& \leqslant C\left(\sum_{j<n} E\left\|V_{j}^{\prime}-E V_{j}^{\prime}\right\|^{q}\right)^{p / q}+C \sum_{j<n} E\left\|V_{j}^{\prime \prime}\right\|^{p} \\
& \leqslant C\left(|n| M^{q}\right)^{p / q}+C|n| \epsilon \\
& =o(|n|), \quad \text { as }|n| \longrightarrow \infty .
\end{aligned}
$$

While the proof of Theorem 2.1 and the proof of the main result in Thanh [11] are similar, we now show in Theorem 2.2 that the implication $((2.1) \Rightarrow(2.2))$ in Theorem 2.1 indeed completely characterizes stable-type $p$ Banach spaces. 
Theorem 2.2. Let $1 \leqslant p<2$ and let $\mathscr{X}$ be a real separable Banach space. Then the following statements are equivalent.

(i) $\mathscr{X}$ is of stable-type $p$.

(ii) For every $d$-dimensional array $\left\{V_{n}, n \in \mathbb{Z}_{+}^{d}\right\}$ of independent mean-zero random elements in $\mathscr{X}$, the condition (2.1) implies (2.2).

(iii) For every d-dimensional array $\left\{V, V_{n}, n \in \mathbb{Z}_{+}^{d}\right\}$ of independent mean-zero random elements in $\mathscr{X}$, the conditions

$$
E\|V\|^{p}<\infty, \quad \sup _{n \in \mathbb{Z}_{+}^{d}} P\left\{\left\|V_{n}\right\|>t\right\} \leqslant C P\{\|V\|>t\}, \quad \forall t>0
$$

imply (2.2).

Proof. The implication $((\mathrm{i}) \Rightarrow(\mathrm{ii}))$ is precisely Theorem 2.1 , whereas the implication $((\mathrm{ii}) \Rightarrow($ iii $))$ is immediate. It remains to verify the implication $(($ iii $) \Rightarrow(\mathrm{i}))$. For reasons of clarity, we collect some of the steps in the following lemmas. The first lemma is a slight modification of de Acosta [4, Theorem 3.1] which holds for sequences of independent identically distributed random elements. The proof of the following modification can be obtained from de Acosta [4, Theorem 3.1] line by line, and so will be omitted.

Lemma 2.3. Let $\mathscr{X}$ be a real separable Banach space, $1 \leqslant p<2$. Let $\left\{V, W_{k}, k \geq 1\right\}$ be sequence of independent random elements such that $E\|V\| p<\infty$ and $\sup _{k \geqslant 1} P\left\{\left\|W_{k}\right\|>\right.$ $t\} \leqslant C P\{\|V\|>t\}$ for all $t>0$. Then

$$
\lim _{n \rightarrow \infty} \frac{\sum_{k=1}^{n} W_{k}}{n^{1 / p}}=0 \quad \text { in probability }
$$

if and only if

$$
\lim _{n \rightarrow \infty} \frac{\sum_{k=1}^{n} W_{k}}{n^{1 / p}}=0 \quad \text { a.s. }
$$

Lemma 2.4. Let $1 \leqslant p<2$ and let $\mathscr{X}$ be a real separable Banach space. Suppose that for every sequence $\left\{V, W_{k}, k \geqslant 1\right\}$ of independent mean-zero random elements in $\mathscr{X}$, the conditions

$$
E\|V\|^{p}<\infty, \quad \sup _{k \geqslant 1} P\left\{\left\|W_{k}\right\|>t\right\} \leqslant C P\{\|V\|>t\}, \quad \forall t>0,
$$

imply that

$$
\lim _{n \rightarrow \infty} \frac{\sum_{k=1}^{n} W_{k}}{n^{1 / p}}=0 \quad \text { in probability. }
$$

Then $\mathscr{X}$ is of stable-type $p$.

Proof of Lemma 2.4. Let $\left\{\varepsilon_{k}, k \geqslant 1\right\}$ be a Rademacher sequence and let $\left\{x_{k}, k \geqslant 1\right\}$ be a sequence of elements in $\mathscr{L}$ such that

$$
\sup _{k \geqslant 1}\left\|x_{k}\right\|<\infty
$$


Then by the hypothesis of the lemma,

$$
\lim _{n \rightarrow \infty} \frac{\sum_{k=1}^{n} \varepsilon_{k} x_{k}}{n^{1 / p}}=0 \quad \text { in probability. }
$$

By Lemma 2.3,

$$
\lim _{n \rightarrow \infty} \frac{\sum_{k=1}^{n} \varepsilon_{k} x_{k}}{n^{1 / p}}=0 \quad \text { a.s. }
$$

Hence, by Theorem 1.2, $\mathscr{X}$ is of stable-type $p$. The proof of Lemma 2.4 is completed.

We now prove the implication ((iii) $\Rightarrow(\mathrm{i}))$. If $d=1$, then the conclusion follows directly from Lemma 2.4. So, we can assume that $d \geqslant 2$. Let $\left\{V, W_{k}, k \geqslant 1\right\}$ be a sequence of independent mean-zero random elements in $\mathscr{X}$ such that $E\|V\| P<\infty$ and $\sup _{k \geqslant 1} P\left\{\left\|W_{k}\right\|>\right.$ $t\} \leqslant C P\{\|V\|>t\}$ for all $t>0$. For $n=\left(n_{1}, \ldots, n_{d}\right) \in \mathbb{Z}_{+}^{d}$, set

$$
\begin{aligned}
& V_{\left(n_{1}, \ldots, n_{d}\right)}=W_{n_{1}}, \quad \text { if } n_{2}=\cdots=n_{d}=1, \\
& V_{\left(n_{1}, \ldots, n_{d}\right)}=0, \quad \text { if } \max \left\{n_{2}, \ldots, n_{d}\right\} \geqslant 2 .
\end{aligned}
$$

Then $\left\{V_{n}, n \in \mathbb{Z}_{+}^{d}\right\}$ is an array of independent mean-zero random elements, and

$$
\sup _{n \in \mathbb{Z}_{+}^{d}} P\left\{\left\|V_{n}\right\|>t\right\} \leqslant C P\{\|V\|>t\}, \quad \forall t>0 .
$$

By (iii),

$$
\frac{1}{|n|^{1 / p}} \sum_{j<n} V_{j} \longrightarrow 0 \quad \text { in } L^{p} \text { as }|n| \longrightarrow \infty .
$$

This implies by taking $n_{2}=\cdots=n_{d}=1$ and letting $n_{1} \rightarrow \infty$ that

$$
\frac{1}{n_{1}^{1 / p}} \sum_{k=1}^{n_{1}} W_{k} \longrightarrow 0 \quad \text { in } L^{p} \text {, so in probability as } n_{1} \longrightarrow \infty \text {. }
$$

By Lemma 2.4, $\mathscr{X}$ is of stable-type $p$.

Remark 2.5. In Theorem 2.1, if $0<p<1$, then the independence hypothesis and the hypothesis that the $\left\{V_{n}, n \in \mathbb{Z}_{+}^{d}\right\}$ have mean-zero are not needed for the theorem to hold.

Indeed, for arbitrary $\epsilon>0$, define $V_{n}^{\prime}$ and $V_{n}^{\prime \prime}, n \in \mathbb{Z}_{+}^{d}$ as in the proof of Theorem 2.1. If $0<p<1$, then

$$
\begin{aligned}
E\left\|\sum_{j<n} V_{j}\right\|^{p} & \leqslant E\left\|\sum_{j<n} V_{j}^{\prime}\right\|^{p}+E\left\|\sum_{j<n} V_{j}^{\prime \prime}\right\|^{p} \\
& \leqslant E\left\|\sum_{j<n} V_{j}^{\prime}\right\|^{p}+\sum_{j<n} E\left\|V_{j}^{\prime \prime}\right\|^{p} \\
& \leqslant(|n| M)^{p}+|n| \epsilon \\
& =o(|n|), \quad \text { as }|n| \longrightarrow \infty .
\end{aligned}
$$




\section{Acknowledgments}

The author is grateful to the referees for carefully reading the manuscript and for offering some very perceptive comments which helped him to improve the paper. The author is grateful to Professor Andrew Rosalsky (University of Florida, USA) for some helpful discussions and for $[4,5,9,12]$. This paper was supported in part by the National Basic Research Program in Natural Science, Vietnam.

\section{References}

[1] A. Adler, M. O. Cabrera, A. Rosalsky, and A. I. Volodin, Degenerate weak convergence of row sums for arrays of random elements in stable type $p$ Banach spaces, Bulletin of the Institute of Mathematics. Academia Sinica 27 (1999), no. 3, 187-212.

[2] A. Adler, A. Rosalsky, and A. I. Volodin, A mean convergence theorem and weak law for arrays of random elements in martingale type p Banach spaces, Statistics \& Probability Letters 32 (1997), no. $2,167-174$.

[3] M. O. Cabrera and A. I. Volodin, Mean convergence theorems and weak laws of large numbers for weighted sums of random variables under a condition of weighted integrability, Journal of Mathematical Analysis and Applications 305 (2005), no. 2, 644-658.

[4] A. de Acosta, Inequalities for B-valued random vectors with applications to the strong law of large numbers, The Annals of Probability 9 (1981), no. 1, 157-161.

[5] A. Gut, Marcinkiewicz laws and convergence rates in the law of large numbers for random variables with multidimensional indices, The Annals of Probability 6 (1978), no. 3, 469-482.

[6] V. Mandrekar and J. Zinn, Central limit problem for symmetric case: convergence to non-Gaussian laws, Studia Mathematica 67 (1980), no. 3, 279-296.

[7] M. B. Marcus and W. A. Woyczyński, Stable measures and central limit theorems in spaces of stable type, Transactions of the American Mathematical Society 251 (1979), 71-102.

[8] G. Pisier, Probabilistic methods in the geometry of Banach spaces, Probability and Analysis (Varenna, 1985), Lecture Notes in Mathematics, vol. 1206, Springer, Berlin, 1986, pp. 167-241.

[9] A. Rosalsky and M. Sreehari, $L_{r}$ convergence theorems with or without random indices for randomly weighted sums of random elements in martingale type p Banach spaces, Stochastic Modelling and Applications 4 (2001), 1-15.

[10] A. Rosalsky, M. Sreehari, and A. I. Volodin, Mean convergence theorems with or without random indices for randomly weighted sums of random elements in Rademacher type p Banach spaces, Stochastic Analysis and Applications 21 (2003), no. 5, 1169-1187.

[11] L. V. Thanh, On the $L^{p}$-convergence for multidimensional arrays of random variables, International Journal of Mathematics and Mathematical Sciences 2005 (2005), no. 8, 1317-1320.

[12] D. Wei and R. L. Taylor, Convergence of weighted sums of tight random elements, Journal of Multivariate Analysis 8 (1978), no. 2, 282-294.

[13] W. A. Woyczyński, Geometry and martingales in Banach spaces. II: independent increments, Probability on Banach Spaces (J. Kuelbs and P. Ney, eds.), Advances in Probability and Related Topics, vol. 4, Marcel Dekker, New York, 1978, pp. 267-517.

Le Van Thanh: Department of Mathematics, Vinh University, Nghe An 42118, Vietnam

E-mail address: lvthanhvinh@yahoo.com 


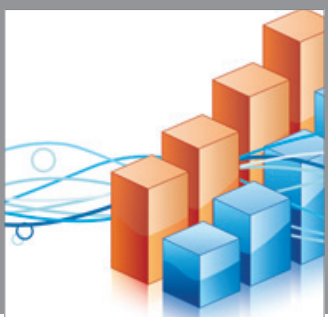

Advances in

Operations Research

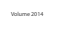

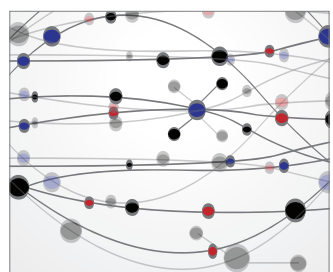

\section{The Scientific} World Journal
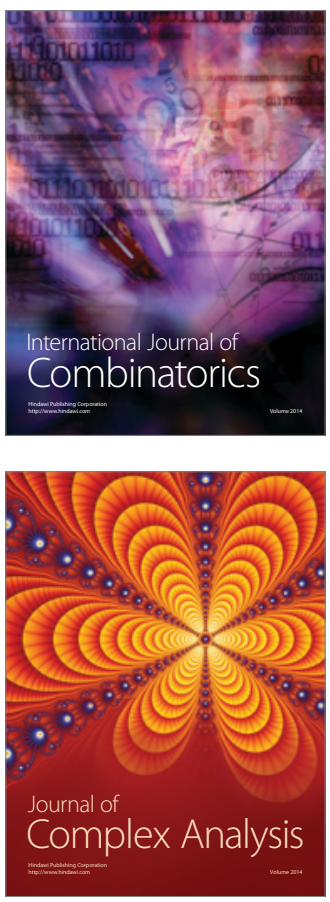

International Journal of

Mathematics and

Mathematical

Sciences
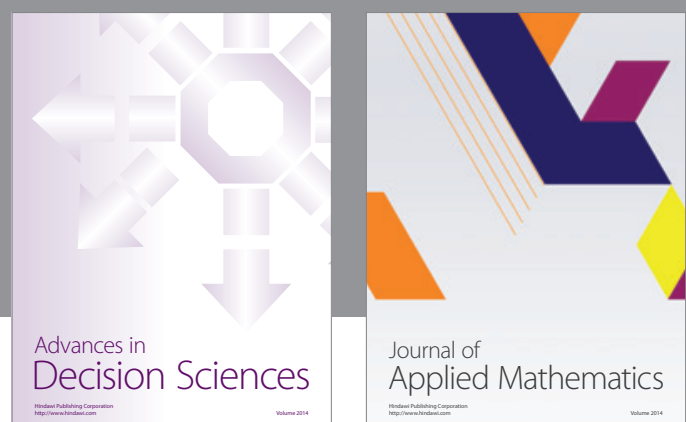

Journal of

Applied Mathematics
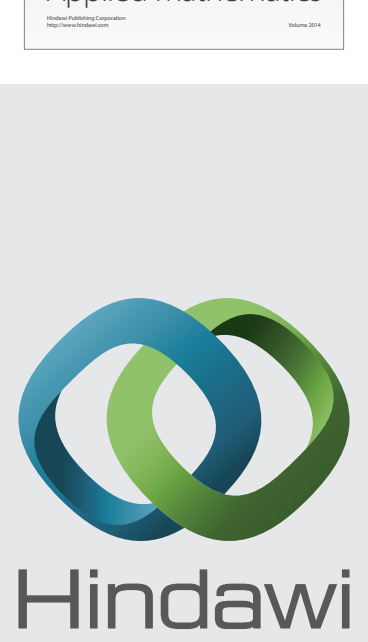

Submit your manuscripts at http://www.hindawi.com
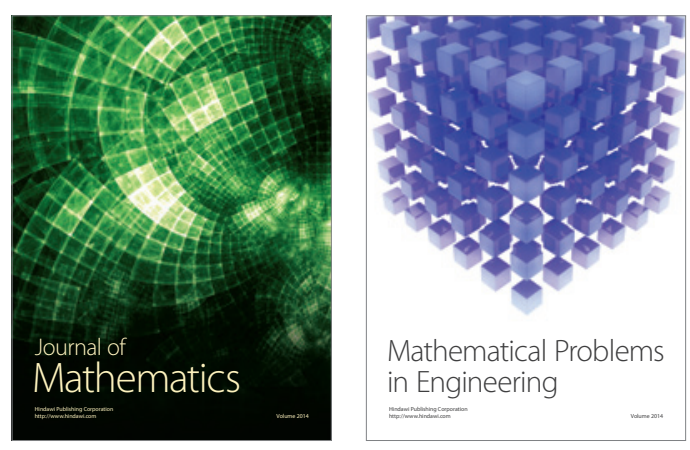

Mathematical Problems in Engineering
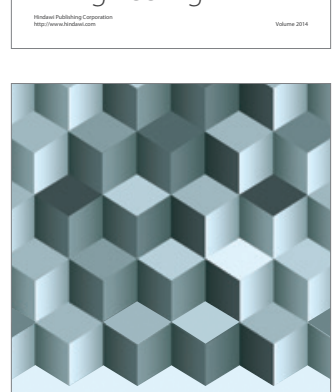

Journal of

Function Spaces
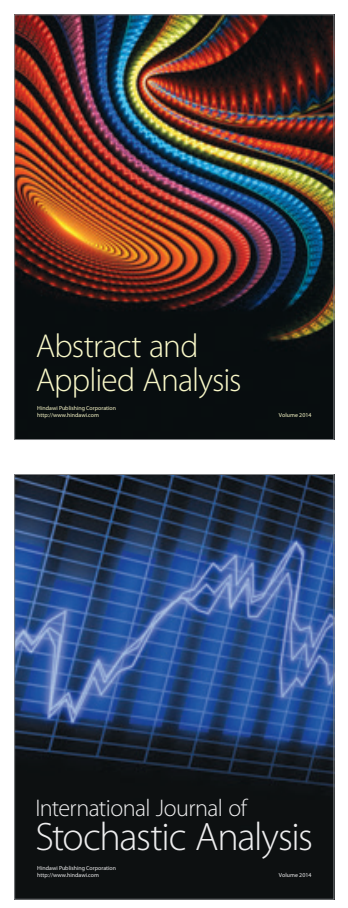

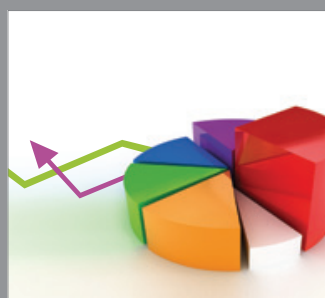

ournal of

Probability and Statistics

Promensencen
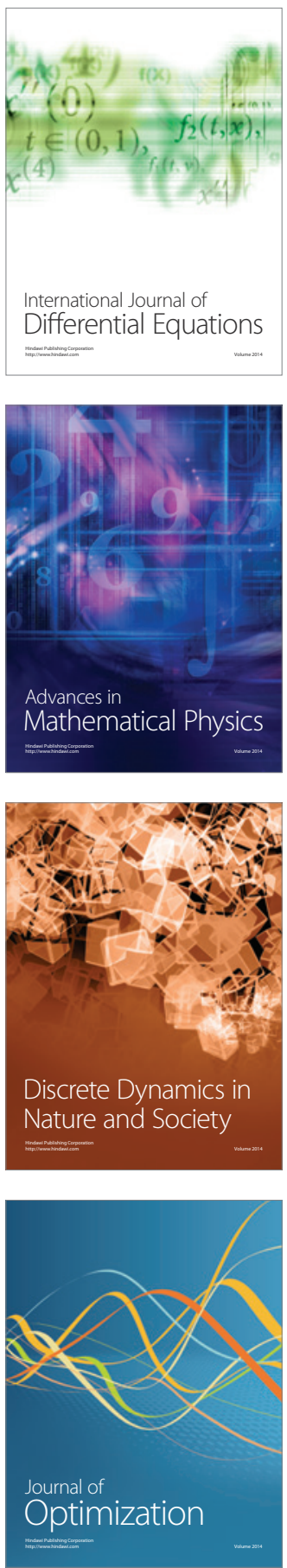